\section{Autoxidation of Catechin}

A mechanism is proposed for the autoxidation of catechin to a polymer (catechin tannin) which has for a long time been recognized to have tanning properties.

Empirical study of the fate of catechin at $100^{\circ}$ has revealed two interesting reaction processes. In agreement with Freudenberg's interpretation ${ }^{1}$ of phlobaphene formation, at $p \mathrm{H}<2$, a buff-coloured phlobaphene, which gave the colour reactions of catechol and phloroglucinol, was rapidly formed both in the presence and absence of oxygen. Between $p H 4$ and $p H$, however, catechin is autoxidized to red catechin tannin. Autoxidation was arrested by sodium hydrogen sulphite, and only epimerization occurred in absence of oxygen. Catechin tannin had phenolic properties, but it lacked the characteristic properties of catechol and phloroglucinol. Under more alkaline conditions another reaction, possibly ringfission ${ }^{2}$ leading to humic acids, intervened.

Catechin in phosphate buffer $(p H$ 8) was shaken at $35^{\circ}$ to constant oxygen uptake; when no catechin could be detected in the paper chromatogram of the reaction mixture, the polymer formed was absorbed on alumina and the hydrogen peroxide in the eluate estimated. The results were: total oxygen uptake 1.59 , hydrogen peroxide formed 0.38 ; oxygen balance, 1.4 moles/mole catechin. Oxygen absorption was represented by a sigmoid curve; but as the rate was slow, namely, 0.0006 mole oxygen/mole catechin/ min., an induction period was not detected.

When the autoxidation of catechin in phosphate buffer was followed spectrophotometrically, no intermediate was detected, and the resulting catechin tannin had $\lambda_{\max } 400 \mathrm{~m} \mu(p \mathrm{H} 6)$ and $\lambda_{\max .} 420-430 \mathrm{~m} \mu$ $(p \mathrm{H}$ 8). Silver oxide oxidation of dioxan solutions of eatechin and eatechol gavo compounds of $\lambda_{\max }$. $370 \mathrm{~m} \mu$ (o-benzoquinone $\left(\mathrm{C}_{4} \mathrm{H}_{8} \mathrm{O}_{2}\right) \lambda_{\max } .370 \mathrm{~m} \mu$ ). Catechin tannin was formed when a solution of the corresponding o-quinone was shaken with oxygen in phosphate buffer, and by silver oxide oxidation of catechin in phosphate buffer.

It is now suggested that the main reaction sequence involves formation of $o$-quinone followed by oxidative condensation. Formation of $o$-quinone probably involves a free radical mechanism, since the reaction takes place in non-polar solvent. Hydrogen peroxide is associated with quinone formation, and its accumulation results from the difference in velocity of the radical processes, conveniently summarized as :

$\mathrm{H}_{2} Q+\mathrm{O}_{2}=Q+\mathrm{H}_{2} \mathrm{O}_{2} ; \mathrm{H}_{2} Q+\mathrm{H}_{2} \mathrm{O}_{2}=Q+2 \mathrm{H}_{2} \mathrm{O}$ The autoxidation of polycyclic hydroquinones has afforded unpublished results (D. E.H.), which confirm formation of hydrogen peroxide in oxidations leading to quinones. Autoxidation of catechin is slow, since the oxidation to quinone is inhibited by phenols ${ }^{3}$. Previous evidence ${ }^{4}$ for intermediate formation of $o$-quinone includes an attempted isolation of a dianilino-derivative (no aralysis or melting point) when tea oxidase acts on catechin. The oxidative condensation is an ionic process requiring dissociating medium, in which addition of phenol to quinone occurs, with CC bond formation between one of the electrophilically activated sites $\left(2^{\prime}, 5^{\prime}, 6^{\prime}\right)$ of the quinone and one of the nucleophilically activated sites $(6,8)$ of another quinone molecule or with the $\left(6,8,2^{\prime}, 5^{\prime}, 6^{\prime}\right)$ sites of a catechin molecule. Addition of phenol to quinone involves transfer of two hydrogen atoms to afford diphenylol, followed by oxidation to the corresponding quinone by means of oxygen, hydrogen peroxide or another molecule of quinone. The oxygen balance agrees with this reaction sequence, which permits the formation of a highly irregular three-dimensional polymer, although certain $\mathrm{CC}$ bonds are excluded on steric considerations. It is significant that since catechol is not bifunctional, it does not undergo a similar polymerization. A number of catechin derivatives are now being synthesized as model substances with which to test these general conclusions. This reaction sequence has also been postulated to explain the formation of melanin ${ }^{5}$ and purpurogallin ${ }^{6}$. It is relevant to a suggestion ${ }^{7}$ for the formation of condensed tannins in Nature. By the action of an oxidase on catechin there results 0 quinone, which is normally reduced by an oxygen acceptor, for example ascorbic acid; but enzymic oxidation of $o$-quinone to catechin tannin occurs whenever the reduction mechanism is intercepted. This explanation is similar to that proposed ${ }^{8}$ for the action of tyrosinase on tyrosine, and the action of plant polyphenolases on catechin is being investigated.

We thank Dr. H. Phillips, director of the British Leather Manufacturers' Research Association, for his advice and encouragement, and the Council of the Association for permission to publish this communication.

\section{E. HATHWAY}

J. W. T. Seakins

British Leather Manufacturers'

Research Association, Milton Park,

Egham, Surrey. March 8.

${ }^{1}$ Freudenberg, K., and Maitland, P., Collegium, 776, 856 (1934).

: Schulze, H., and Flaig, W., Annalen, 575, 231 (1952).

3 James, T. H., Snell, J. M., and Weissberger, A., J. Amer. Chem. Soc., 60,2084 (1938).

4 Lamb, J., and Sreerangachar, H. B., Biochem. J., 34, 1472 (1940). 'Bu'Lock, J. D., and Harley-Mason, J., J. Chem. Soc., 703 (1951).
Beer, R. J. S., Broadhurst, T., and Robertson, A., J. Chem. Soc., 1947 (1954). - Critchlow, A., Haworth, R. D., and Pauson, P. L., J. Chem. Soc.,

"Houghton Roberts, E. A., "Adv. Enzymol.", 2, 113 (1942).

'Robinson, E. S., and Nelson, J. M., Arch. Biochem. Biophys., 4, 111 (1944).

\section{Occurrence of Different Beta-Lactoglobulins in Cow's Milk}

DURING the past twenty years, numerous physicochemical investigations of crystalline $\beta$-lactoglobulin prepared from cow's milk have revealed a degree of heterogeneity puzzling in a protein considered to be 'pure' (see the recent discussion of this problem by Ogston and Tilley'). In general, mixed milk has been used as the starting material, and it has been tacitly assumed that the $\beta$-lactoglobulin synthesized by the mammary gland is the same for all cows. This is not the case.

When a suitably prepared fraction of the milk of single cows (for example, the proteins soluble in 20 per cent sodium sulphate) was subjected to electrophoresis on filter-paper in barbitone buffer at $p \mathrm{H} 8 \cdot 6$, $I=0 \cdot 05$, using an apparatus of the type described by Flynn and de $\mathrm{Mayo}^{2}$, and applying $230 \mathrm{~V}$. for $16 \mathrm{hr}$., we found that individual cows produce either a mixture of two electrophoretically distinct $\beta$-lactoglobulins or only one or the other of these, as shown in Fig. 1, which gives examples of each type. The two single components will be termed $\beta_{1}$ - and $\beta_{2}$-lactoglobulin, following the nomenclature suggested by Polis, Shmukler, Custer and McMeekin', and the mixture of the two in the milk of an individual cow 